TITLE:

\title{
Improvement on the metric reconstruction scheme in the Regge-Wheeler-Zerilli formalism
}

\author{
$\operatorname{AUTHOR}(\mathrm{S})$ : \\ Jhingan, S; Tanaka, T
}

CITATION:

Jhingan, S ...[et al]. Improvement on the metric reconstruction scheme in the Regge-Wheeler-Zerilli formalism. PHYSICAL REVIEW D 2003,

67(10): 104018.

\section{ISSUE DATE:}

2003-05-15

URL:

http://hdl.handle.net/2433/49999

RIGHT:

Copyright 2003 American Physical Society 
PHYSICAL REVIEW D 67, 104018 (2003)

\title{
Improvement on the metric reconstruction scheme in the Regge-Wheeler-Zerilli formalism
}

\author{
Sanjay Jhingan \\ Yukawa Institute for Theoretical Physics, Kyoto University, Kyoto 606-8502, Japan \\ and Dpto. de Física Teórica, Universidad del País Vasco, Apdo. 644, 48080, Bilbao, Spain \\ Takahiro Tanaka \\ Yukawa Institute for Theoretical Physics, Kyoto University, Kyoto 606-8502, Japan
}

(Received 18 November 2002; published 22 May 2003)

\begin{abstract}
We study master variables in the Regge-Wheeler-Zerilli formalism. We show that a specific choice of new variables is suitable for studying perturbation theory from the viewpoint of radiation reaction calculations. With explicit definition of the improved master variables in terms of components of metric perturbations, we present the master equations, with source terms, and metric reconstruction formulas. In the scheme using these new variables, we do not need any time and radial integrations except for solving the master equation. We also show that the master variable for even parity modes which satisfies the same homogeneous equation as the oddparity case, obtained via Chandrasekhar transformation, does not have the good property in this sense.
\end{abstract}

DOI: 10.1103/PhysRevD.67.104018

PACS number(s): 04.25.Nx

\section{INTRODUCTION}

Black hole perturbation is a powerful tool for the evaluation of gravitational waves from a binary system when its mass ratio is large [1-3]. Although any systematic method to calculate the radiation reaction to the particle motion has not been established so far, there are various new developments in this field [4-17]. A formal prescription to extract the selfforce was developed in [4], generalizing the work of DeWitt and Brehme [18] on the electromagnetic self-force to include the gravitational case. These results were further verified by an independent, and different, axiomatic approach by Quinn and Wald [5]. The prescription can be summarized as follows. The retarded field can be obtained in terms of Green's functions which can be formally decomposed into "direct" and "tail" parts. Roughly speaking, the "direct" part of the field is that part which has support only on the future light cone, emanating from the source point. The "tail" part is composed of contribution due to curvature scattering which pervades inside the future light cone of the source point. The analysis presented in $[4,5]$ indicates that, the particle motion, after taking into account the self-force, follows a geodesic on the geometry perturbed by adding the "tail" part to the original background spacetime.

The actual isolation of the "tail" part is not an easy task. There are ways to calculate the "full" Green's function but there is no direct method to compute the "tail" part alone. Hence, the standard prescription that has emerged in the past few years is to subtract the "direct" part from the "full" metric perturbation. Here lies the well known "gauge problem." In the standard methods for constructing full metric perturbation, we first solve the equation for master variables and then from these master variables we reconstruct the metric perturbations. The result is naturally written in a specific gauge such as Regge-Wheeler (RW) [19,20] or radiation gauge [21-23]. On the other hand, the "direct" part is evaluated in the harmonic gauge associated with the particle trajectory. Therefore, before any meaningful subtraction we need to relate these expressions which are in different gauges. This is by no means an easy task since we do not know the necessary gauge transformation a priori. This additional task to find the appropriate choice of the gauge parameters makes the problem much harder to solve; this is the aforementioned "gauge problem." The attempts for subtraction of the "direct" part in the RW gauge were reported by Mino [6] and Sago et al. [16].

In this paper we would like to revisit the problem of metric perturbations reconstruction from the master variables in case of Schwarzschild background. In this approach, based on Regge-Wheeler-Zerilli formalism, the problem of metric reconstruction is relatively well understood $[19,20]$. What we would like to discuss here are possibilities of improvements on this formalism.

It is well known that in the Schwarzschild case the odd and the even parity perturbations naturally decouple due to spherical symmetry. Assuming a time dependence of the form $\exp (-i \omega t)$, the perturbations of a Schwarzschild black hole can be described by a master equation, for each partial wave mode decomposed in terms of spherical harmonics, as

$$
\frac{d^{2} \zeta}{d r^{* 2}}+\left(\omega^{2}-V\right) \zeta=\mathcal{S}\left(T_{\mu \nu}\right)
$$

Here $\zeta$ is the master variable, $\mathcal{S}$ is the source term composed of the matter energy momentum tensor $T_{\mu \nu}$ and $r^{*}=r$ $+2 M \ln (r / 2 M-1)$ is the usual tortoise radial coordinate. The metric components in the RW gauge, $h^{R W}$, are obtained by applying certain differential operators on the master variable and on the energy-momentum tensor as

$$
h^{R W}=\hat{h}^{(M)}(\zeta)+\hat{h}^{(T)}\left(T_{\mu \nu}\right) .
$$

Then, the formulas for the metric reconstruction in the scheme presented in the original papers $[19,20]$ contain $\omega$ in the denominator of the expressions for $\hat{h}^{(T)}$. Although $\omega$ is just a number in the frequency domain, if in denominator it can be an obstacle in computing the metric in the vicinity of a particle orbiting a black hole. Suppose that the particle 
moves between $r_{\min }$ and $r_{\max }$. The appearance of $\omega$ in the denominator means that $\hat{h}^{(T)}$ is no longer localized on the radial shell where the particle orbit lies. Instead, the source is distributed continuously in the region between $r_{\min }$ and $r_{\max }$. Therefore, the metric components are not completely determined by the notion of the master variables in this region even if the concerned field points are off the shell. In the computation of the self-force, the gravitational field exactly on this shell is unnecessary. A limiting value evaluated along, e.g., the outer radial direction is sufficient for the purpose of computing the self-force. If we can modify the formulation so that $\hat{h}^{(T)}$ is localized on the shell, then we can apply the formula for the metric reconstruction outside the source, which is much simpler. For the even parity case, an improved master variable has already been introduced by Moncrief ([24], see also [25] ${ }^{1}$ ). We give here the general metric reconstruction formulas in the presence of sources, which have not been given explicitly yet, as far as we know. We show that $\omega$ can be removed from the denominator by using Moncrief's master variable. Same argument follows for the odd parity case, i.e., by introducing a new improved master variable, we can remove appearance of $\omega$ in the denominator. Complete expressions for the metric reconstruction are also presented for this case.

Another complication which arises is from the well known fact that the potentials for odd and even parity cases differ from each other. The potential for the even parity case is, relatively, much more complicated. Hence, it would be useful if we could formulate the even parity perturbations to satisfy the same master equation with the odd parity case. Chandrasekhar has already given a unified approach, known as Chandrasekhar transformations, and shown the relation between RW and Zerilli equations [26] (for a comprehensive review see [27]). In this paper we also derive the full metric reconstruction formulas for the even parity perturbation by using the master variable obtained via the Chandrasekhar transformation. Under the requirement for this new master variable to satisfy the RW equation in vacuum, we can still modify its definition by adding a combination of the metric components which appear on the left hand side of Einstein equations, since it is zero in vacuum. Examining all the possibilities of such a modification, we have concluded that we cannot eliminate $\omega$ from the denominator in the expression for $\hat{h}^{(T)}$. Unfortunately, as it turns out, no dramatic simplification happens by reformulating the formulas solely in terms of the variable obtained via Chandrasekhar transformation, although the importance of this transformation is not reduced at all by this fact.

The paper is organized as follows. In Sec. II we discuss the equations for both odd and even parity cases. We have provided the explicit expressions for source terms corresponding to the new master variables. In Sec. III the even parity master variable which satisfies odd parity homoge-

\footnotetext{
${ }^{1}$ We thank Dr. Tomohiro Harada for informing us about this reference. After finishing our paper, we noticed that the new variable for odd parity case was also introduced in this reference before.
}

neous master equation is discussed. We briefly summarize the results obtained in this paper in Sec. IV, with a speculation towards an alternative method to compute the regularized self-force subtracting the direct part at the level of the master variables.

\section{IMPROVED MASTER VARIABLES}

We begin with reexamining the Regge-Wheeler-Zerilli (RW) formulation. In this formalism a master equation for a master variable is derived, which are called the RW equation and the RW variable, respectively. Once we know the solution for the RW variable, all the components of the metric perturbation can be derived from it. This scheme is well known. What we have shown here is that it can be improved, in the sense discussed earlier, by introducing alternative master variables.

We consider the Schwarzschild metric,

$$
\begin{aligned}
d s^{2}= & -\left(1-\frac{2 M}{r}\right) d t^{2}+\left(1-\frac{2 M}{r}\right)^{-1} d r^{2} \\
& +r^{2}\left(d \theta^{2}+\sin ^{2} \theta d \varphi^{2}\right)
\end{aligned}
$$

as the background. The 10 metric components can be decomposed into "odd" and "even" parity modes. We use the notation in which, after harmonic decomposition for the angular dependence, $H_{0}, H_{1}, H_{2}, h_{0}^{(e)}, h_{1}^{(e)}, K$ and $G$ are the components of metric perturbations for the even parity modes, and $h_{0}, h_{1}$ and $h_{2}$ are for the odd parity modes. Here we assume that the time dependence is given by $\exp (-i \omega t)$. Similarly, the components of the energy momentum tensor can be decomposed into odd and even parity modes. $A^{(0)}, A^{(1)}, A, B^{(0)}, B, G^{(s)}$ and $F$ are the expansion coefficients for the even parity modes, and $Q^{(0)}, Q$ and $D$ are for the odd parity modes (we follow throughout notation of Zerilli for the metric perturbation and the energy-momentum tensor with slight modifications; see [16] for the basic equations such as the law of gauge transformation and the definitions of the harmonic expansion coefficients of the energy momentum tensor).

\section{A. Odd parity}

First, we consider the odd parity case. The RW gauge choice corresponds to setting $h_{2}^{R W}=0$. Here, the variables with a superscript $R W$ means the quantities are in the RW gauge. The nontrivial set of Einstein equations for the odd parity mode is

$$
\begin{aligned}
h_{0, r r}^{R W} & +i \omega h_{1, r}^{R W}+i \omega \frac{2}{r} h_{1}^{R W}+\left[\frac{4 M}{r}-2(1+\lambda)\right] \frac{h_{0}^{R W}}{r(r-2 M)} \\
& =\frac{8 \pi}{\sqrt{1+\lambda}} \frac{r^{2}}{r-2 M} Q^{(0)}
\end{aligned}
$$




$$
\begin{gathered}
-\omega^{2} h_{1}^{R W}+i \omega h_{0, r}^{R W}+2 \lambda(r-2 M) \frac{h_{1}^{R W}}{r^{3}}-i \omega \frac{2}{r} h_{0}^{R W} \\
=-\frac{8 \pi i}{\sqrt{1+\lambda}}(r-2 M) Q, \\
\left(1-\frac{2 M}{r}\right) h_{1, r}^{R W}+i \omega\left(1-\frac{2 M}{r}\right)^{-1} h_{0}^{R W}+\frac{2 M}{r^{2}} h_{1}^{R W} \\
=-\frac{8 \pi i}{\sqrt{2 \lambda(1+\lambda)}} r^{2} D .
\end{gathered}
$$

From the above equations, and using the conventional gauge invariant master variable ${ }^{(o)} \chi$

$$
{ }^{(o)} \chi=\frac{r-2 M}{r^{2}} h_{1}^{R W}
$$

we can derive a second order differential equation as

$$
\left[\partial_{r^{*}}^{2}+\omega^{2}-V^{R W}(r)\right]^{(o)} \chi=\mathcal{S}^{(o)} \chi .
$$

This is the well known Regge-Wheeler equation [19]. Here

$$
V^{R W}=\left(1-\frac{2 M}{r}\right)\left(\frac{2(\lambda+1)}{r^{2}}-\frac{6 M}{r^{3}}\right),
$$

is the Regge-Wheeler potential and the source term is given by

$$
\begin{aligned}
\mathcal{S}^{(o)} \chi= & \frac{8 \pi i}{\sqrt{\lambda+1}}\left(1-\frac{2 M}{r}\right)\left[\left(1-\frac{2 M}{r}\right) Q\right. \\
& \left.+\frac{r}{\sqrt{2 \lambda}} \partial_{r}\left(\frac{r-2 M}{r} D\right)\right] .
\end{aligned}
$$

Here $\lambda$ is defined in terms of $\ell$, the total angular momentum of the spherical harmonics, as

$$
\lambda \equiv \frac{(\ell-1)(\ell+2)}{2} .
$$

Once the master variable and the energy-momentum tensor are given, we can reconstruct the metric perturbations. To distinguish these reconstructed variables from the original ones, we associate them with an overhat in the same way as $\hat{h}^{(M)}$ and $\hat{h}^{(T)}$. Combining the Einstein equations using the definition of the master variable, the necessary formulas for the reconstruction can be derived as

$\hat{h}_{1}^{R W}={\frac{r^{2}}{r-2 M}}^{(o)} \chi$

$$
\begin{aligned}
\hat{h}_{0}^{R W}= & -\frac{1}{i \omega}\left(1-\frac{2 M}{r}\right)\left[\left(r^{(o)} \chi\right)_{, r}\right. \\
& \left.+\frac{8 \pi i r^{2}}{\sqrt{2 \lambda(\lambda+1)}} D\right] .
\end{aligned}
$$

For $h_{1}^{R W}$ reconstruction is straightforward since there is only $\hat{h}^{(M)}$. In reconstructed $h_{0}^{R W}$ first term in the square brackets corresponds to $\hat{h}^{(M)}$ and second term is $\hat{h}^{(T)}$. The trouble with the expression for $\hat{h}^{(T)}$ is the presence of $\omega$ in denominator, as anticipated earlier. Even if we rewrite this expression using conservation law

$$
\sqrt{2 \lambda} D=\frac{\omega r^{2}}{r-2 M} Q^{(0)}+\left(3-\frac{4 M}{r}\right) Q+(r-2 M) Q_{, r},
$$

this $\omega$ cannot be removed. This fact implies that we need time integration of the source term in the reconstruction of metric perturbation. Hence the reconstructed metric is not solely determined by the master variable even when the energy-momentum tensor vanishes on the spherical shell containing a given field point.

We, therefore, introduce a new gauge invariant variable ${ }^{(o)} \zeta$ defined by

$$
{ }^{(o)} \zeta=-\frac{r}{2 \lambda}\left[-i \omega h_{1}^{R W}-h_{0, r}^{R W}+\frac{2}{r} h_{0}^{R W}\right] .
$$

Using the definition (2.5) with one of the odd-parity field equations, we can verify that

$$
-i \omega^{(o)} \zeta={ }^{(o)} \chi+\frac{8 \pi i r(r-2 M) Q}{2 \lambda \sqrt{1+\lambda}} .
$$

Hence, ${ }^{(o)} \zeta$ is equivalent to time integral of the original variable ${ }^{(o)} \chi$ outside the source distribution. The expression applicable to an arbitrary gauge has the same functional form as in the RW gauge:

$$
{ }^{(o)} \zeta=-\frac{r}{2 \lambda}\left[-i \omega h_{1}-h_{0, r}+\frac{2}{r} h_{0}\right] \text {. }
$$

Substituting Eq. (2.13) into Eq. (2.6), we recover

$$
\left[\partial_{r^{*}}^{2}+\omega^{2}-V^{R W}(r)\right]^{(o)} \zeta=\mathcal{S}^{(o)} \zeta
$$

with new source term

$$
\mathcal{S}^{(o)} \zeta=\frac{8 \pi(r-2 M)}{2 \lambda \sqrt{1+\lambda}}\left[\omega r Q-\partial_{r}\left(r Q^{(0)}\right)\right] .
$$

Here we have used conservation law (2.11) to simplify the expression. The source term $\mathcal{S}^{(o)} \zeta$ does not have a time integral although ${ }^{(o)} \zeta$ is a time integral of the original variable ${ }^{(o)} \chi$. This is expected a priori. If the source term for ${ }^{(o)} \zeta$ has an integration constant then it is not uniquely determined, which contradicts the fact that it is a gauge invariant 
variable. Here, in order to illustrate the way how we found the new variable, we took rather lengthy steps to obtain the master equation (2.15) passing through the equation for the original master variable (2.6). But, of course, one can directly verify the final result by combining first two oddparity equations (2.2) and (2.3).

Now we consider the reconstruction of the metric components from this master variable ${ }^{(o)} \zeta$. There are two nonvanishing components $h_{0}^{R W}$ and $h_{1}^{R W}$ in the RW gauge. They are to be solely determined from ${ }^{(o)} \zeta$, if the metric perturbation satisfies Einstein equations. From Eq. (2.3) and the definition of ${ }^{(o)} \zeta$, we immediately have

$$
\hat{h}_{1}^{R W}=-{\frac{i \omega r^{2}}{r-2 M}}^{(o)} \zeta+\frac{4 \pi i r^{3}}{\lambda \sqrt{1+\lambda}} Q .
$$

Once we know $\hat{h}_{1}^{R W}$, we can reconstruct $\hat{h}_{0}^{R W}$ by using Eq. (2.4) as

$$
\hat{h}_{0}^{R W}=(r-2 M)\left({ }^{(o)} \zeta_{, r}+\frac{1}{r}{ }^{(o)} \zeta+\frac{4 \pi r^{2}}{\lambda \sqrt{1+\lambda}} Q^{(0)}\right) .
$$

This time, the $\hat{h}^{(T)}$ part does not have $\omega$ in the denominator. Therefore, one can simply set the source terms to zero to obtain the formulas for the reconstruction of the metric perturbation in vacuum region. We notice here that $\hat{h}^{(M)}$ is also free from annoying factor $\omega$. These two facts are actually related. By definition, the defining expression for a gauge invariant master variable does not have $\omega$ in the denominator. Otherwise, the gauge invariant variable would be ambiguous due to integration constant, and information of metric perturbations in the vicinity of a spherical shell, specified by $t$ and $r$, will be insufficient to determine the corresponding gauge invariant variable there. Let us assume that $\hat{h}^{(M)}$ also does not have $\omega$ in the denominator. In the vacuum case, we can consider a cycle of operations starting with $h$, going through the master variable, and again coming back to $h$ by using $\hat{h}^{(M)}$. Throughout this cycle, there is no $\omega$ in the denominator. Hence, if a homogeneous solution of metric perturbations including its derivatives near a spherical shell is given, this cycle should reproduce the original metric perturbations. The formulas composing this cycle will not change even if there exists matter source away from the spherical shell. If $\hat{h}^{(T)}$ has $\omega$ in the denominator, this term gives an additional contribution even if matter source does not exist there. This is a contradiction. Hence, if $\hat{h}^{(M)}$ does not have $\omega$ in the denominator, neither does $\hat{h}^{(T)}$.

\section{B. Even parity}

Next, we look at the even parity case. The RW gauge choice corresponds to setting $h_{0}^{(e) R W}=h_{1}^{(e) R W}=G^{R W}=0$. The set of field equations for the even parity modes, with the source terms, is

$$
\begin{aligned}
& \left(1-\frac{2 M}{r}\right)\left[\left(1-\frac{2 M}{r}\right)\left(K_{, r r}^{R W}-\frac{1}{r} H_{2, r}^{R W}\right)+\left(3-\frac{5 M}{r}\right) \frac{1}{r} K_{, r}^{R W}\right. \\
& \left.-\frac{1}{r^{2}}\left(H_{2}^{R W}-K^{R W}\right)-\frac{\lambda}{r^{2}}\left(H_{2}^{R W}+K^{R W}\right)\right]=-8 \pi A^{(0)}, \\
& -i \omega K_{, r}^{R W}-i \omega \frac{1}{r}\left(K^{R W}-H_{2}^{R W}\right)+i \omega \frac{M}{r(r-2 M)} K^{R W} \\
& -\frac{(1+\lambda)}{r^{2}} H_{1}^{R W}=-\frac{8 \pi i}{\sqrt{2}} A^{(1)}, \\
& \frac{1}{(r-2 M)}\left[-\omega^{2} \frac{r^{2}}{(r-2 M)} K^{R W}-\left(1-\frac{M}{r}\right) K_{, r}^{R W}+2 i \omega H_{1}^{R W}\right. \\
& +\frac{(r-2 M)}{r} H_{0, r}^{R W}+\frac{1}{r}\left(H_{2}^{R W}-K^{R W}\right) \\
& \left.+\frac{(1+\lambda)}{r}\left(K^{R W}-H_{0}^{R W}\right)\right] \\
& =-8 \pi A \text {, } \\
& {\left[\left(1-\frac{2 M}{r}\right) H_{1}^{R W}\right]_{, r}+i \omega\left(H^{R W}+K^{R W}\right)=\frac{8 \pi i}{\sqrt{1+\lambda}} r B^{(0)},} \\
& i \omega H_{1}^{R W}+\left(1-\frac{2 M}{r}\right)\left(H_{0}^{R W}-K^{R W}\right)_{, r}+\frac{2 M}{r^{2}} H_{0}^{R W}+\frac{1}{r}\left(1-\frac{M}{r}\right) \\
& \times\left(H_{2}^{R W}-H_{0}^{R W}\right)=\frac{8 \pi}{\sqrt{1+\lambda}}(r-2 M) B, \\
& \omega^{2}\left(1-\frac{2 M}{r}\right)^{-1}\left(K^{R W}+H_{2}^{R W}\right)+\left(1-\frac{2 M}{r}\right)\left[K_{, r r}^{R W}-H_{0, r r}^{R W}\right] \\
& +\left(1-\frac{M}{r}\right) \frac{2}{r} K_{, r}^{R W}-2 i \omega H_{1, r}^{R W}-i \omega \frac{2(r-M)}{r(r-2 M)} H_{1}^{R W} \\
& -\frac{1}{r}\left(1-\frac{M}{r}\right) H_{2, r}^{R W}-\frac{1}{r}\left(1+\frac{M}{r}\right) H_{0, r}^{R W} \\
& -\frac{(1+\lambda)}{r^{2}}\left(H_{2}^{R W}-H_{0}^{R W}\right) \\
& =8 \sqrt{2} \pi G^{(s)}, \\
& H_{0}^{R W}-H_{2}^{R W}=\frac{16 \pi}{\sqrt{2 \lambda(1+\lambda)}} r^{2} F .
\end{aligned}
$$

The original Zerilli's master variable, $R_{l m}$, is defined by

$$
R_{l m}=\frac{1}{\omega}{ }^{(e)} \chi=\frac{1}{i \omega}\left(\frac{r-2 M}{\lambda r+3 M}\right)\left[\frac{i \omega r^{2}}{r-2 M} K^{R W}+H_{1}^{R W}\right],
$$


and has an ambiguity due to an integration constant. We will work, instead, with its gauge invariant form ${ }^{(e)} \chi$. With the field equations above it obeys the wave equation [20]

$$
\left.\left[\partial_{r *}^{2}+\omega^{2}-V^{Z}(r)\right]^{(e)} \chi=\mathcal{S}^{(e)} \chi\right) .
$$

Here,

$$
\begin{aligned}
V^{Z}(r)= & \left(1-\frac{2 M}{r}\right) \\
& \times \frac{2 \lambda^{2}(\lambda+1) r^{3}+6 \lambda^{2} M r^{2}+18 \lambda M^{2} r+18 M^{3}}{r^{3}(r \lambda+3 M)^{2}}
\end{aligned}
$$

is the Zerilli potential and the source term takes the form

$$
\begin{aligned}
\left.S^{(e)} \chi\right)= & \frac{(r-2 M)^{2}}{(r \lambda+3 M) \sqrt{1+\lambda}} B_{, r}^{(0)} \\
& +\frac{(r-2 M)\left(-12 M^{2}+9 M r+r^{2} \lambda\right)}{r \sqrt{1+\lambda}(r \lambda+3 M)^{2}} B^{(0)} \\
& -\sqrt{2} \lambda \frac{(r-2 M)^{2}}{(r \lambda+3 M)^{2}} A^{(1)}+\omega\left[-\frac{r^{2}}{(r \lambda+3 M)} A^{(0)}\right. \\
& +\frac{(r-2 M)^{2}}{(r \lambda+3 M)} A+\frac{(r+2 M)^{2}}{(r \lambda+3 M) \sqrt{1+\lambda}} B \\
& \left.-\sqrt{2} \frac{(r-2 M)}{\sqrt{\lambda(1+\lambda)}} F\right]
\end{aligned}
$$

The formulas for the metric reconstruction are derived by combining the Einstein equations using the definition of the master variable. Since this is a known result, we just quote here the explicit reconstruction formula for $K^{R W}$ as an example:

$$
\begin{aligned}
\hat{K}^{R W}= & \frac{1}{\omega}\left[-\left(1-\frac{2 M}{r}\right){ }^{(e)} \chi_{, r}\right. \\
& \left.+\frac{r^{2} \lambda+(r \lambda+3 M)(r \lambda+2 M)}{r^{2}(r \lambda+3 M)} \chi\right] \\
& -\frac{r(r-2 M)}{\omega(r \lambda+3 M)}\left(\frac{1}{\sqrt{2}} A^{(1)}+\frac{1}{\sqrt{1+\lambda}} B^{(0)}\right) .
\end{aligned}
$$

As in the odd parity case, the first term is $\hat{h}^{(M)}$ and the second term is $\hat{h}^{(T)}$. The presence of $\omega$ in denominator in the expression for $\hat{h}^{(T)}$ is a signal that this ${ }^{(e)} \chi$ is not the most convenient choice of the master variable.

Analogous to the odd parity case we now define a new time integrated variable using vacuum field equations as

$$
\text { (e) } \zeta=\frac{r(r-2 M)}{(\lambda+1)(\lambda r+3 M)}\left[H_{2}^{R W}-r K_{, r}^{R W}+\frac{r \lambda+3 M}{r-2 M} K^{R W}\right] \text {. }
$$

In fact, the same variable has been introduced earlier by Moncrief [24] (see also Gleiser et al. [28]). It can be easily checked that ${ }^{(e)} \zeta$ satisfies a similar wave equation

$$
\left[\partial_{r *}^{2}+\omega^{2}-V^{Z}(r)\right]^{(e)} \zeta=\mathcal{S}^{(e)} \zeta,
$$

with a modified source term

$$
\begin{aligned}
\mathcal{S}^{(e)} \zeta= & \frac{r-2 M}{(1+\lambda)(r \lambda+3 M)}\left[r^{2} A_{, r}^{(0)}-r\left(\frac{r \lambda+2 M}{r-2 M}\right.\right. \\
& \left.-\frac{r \lambda+9 M}{r \lambda+3 M}\right) A^{(0)}-\omega \frac{r^{2}}{\sqrt{2}} A^{(1)}+(1+\lambda)(r-2 M) A \\
& \left.+\sqrt{1+\lambda}(r-2 M) B-\sqrt{\frac{2(1+\lambda)}{\lambda}}(r \lambda+3 M) F\right] .
\end{aligned}
$$

Here, for simplification, we have used the three constraint equations, corresponding to $T_{\nu ; \mu}^{\mu}=0$, which are

$$
\begin{aligned}
A_{, r}^{(1)}= & \frac{1}{r-2 M}\left[-\frac{\sqrt{2} \omega r^{2}}{r-2 M} A^{(0)}-2\left(1-\frac{M}{r}\right) A^{(1)}\right. \\
& +\sqrt{\left.2(1+\lambda) B^{(0)}\right]} \\
A_{, r}= & \frac{1}{r-2 M}\left[\frac{\omega r^{2}}{\sqrt{2}(r-2 M)} A^{(1)}-\frac{M r}{(r-2 M)^{2}} A^{(0)}\right. \\
& \left.+\frac{M-2 r}{r} A+\sqrt{1+\lambda} B+\sqrt{2} G^{(s)}\right], \\
B_{, r}= & \frac{1}{r-2 M}\left[\frac{\omega r^{2}}{r-2 M} B^{(0)}-\left(3-\frac{4 M}{r}\right) B+\sqrt{2 \lambda} F\right. \\
& \left.-\sqrt{2(1+\lambda)} G^{(s)}\right] .
\end{aligned}
$$

As explained in the odd parity case, the source term for a gauge invariant variable does not have $\omega$ in denominator. Now we come to the reconstruction of the metric components using this new master variable ${ }^{(e)} \zeta$ in the RW gauge. There are four nonvanishing components in the even parity case, namely, $K^{R W}, H_{1}^{R W}, H_{0}^{R W}$ and $H_{2}^{R W}$. We can rewrite them in terms of the gauge invariant variable ${ }^{(e)} \zeta$ as

$$
\begin{aligned}
\hat{K}^{R W}= & \frac{\lambda(\lambda+1) r^{2}+3 \lambda M r+6 M^{2}}{r^{2}(r \lambda+3 M)}(e) \zeta+\left(1-\frac{2 M}{r}\right)(e) \zeta_{, r} \\
& -\frac{8 \pi r^{3}}{(\lambda+1)(r \lambda+3 M)} A^{(0)},
\end{aligned}
$$




$$
\begin{aligned}
& \hat{H}_{1}^{R W}=-i \omega \frac{\lambda r(r-2 M)-M(r \lambda+3 M)}{(r-2 M)(r \lambda+3 M)}{ }^{(e)} \zeta-i \omega r^{(e)} \zeta_{, r} \\
& +i \omega \frac{r^{5}}{(1+\lambda)(r \lambda+3 M)(r-2 M)} A^{(0)} \\
& +i \frac{r^{2}}{\sqrt{2}(1+\lambda)} A^{(1)} \\
& \hat{H}_{2}^{R W}=\frac{1}{r \lambda+3 M}\left[\left(-\omega^{2} r^{2} \frac{(r \lambda+3 M)}{r-2 M}+\lambda^{2}+\frac{3 M^{2}}{r^{2}}\right.\right. \\
& \left.+\frac{\lambda\left(r^{2} \lambda+6 M^{2}\right)}{r(r \lambda+3 M)}\right)(e) \zeta-\left(\frac{M}{r}(r \lambda+3 M)-\lambda(2 M\right. \\
& -r) \mid(e) \zeta_{, r}-\frac{1}{1+\lambda}\left\{\left(\frac{r \lambda}{r \lambda+3 M}-\frac{M}{r-2 M}\right) r^{3} A^{(0)}\right. \\
& +\frac{1}{\sqrt{2}} \omega r^{4} A^{(1)}-(r-2 M) r^{2}[B+(1+\lambda) A] \\
& \left.+\sqrt{\frac{2}{\lambda}} r^{2}(r \lambda+3 M) F\right\}, \\
& \hat{H}_{0}^{R W}=\bar{H}_{2}^{R W}+\frac{16 \pi}{\sqrt{2 \lambda(1+\lambda)}} r^{2} F .
\end{aligned}
$$

These reconstruction formulas are local and do not require any time integrations.

\section{EVEN PARITY MASTER VARIABLE VIA CHANDRASEKHAR TRANSFORMATION}

In this section we have examined the even parity master variable that satisfies the same homogeneous master equation as the odd parity one. The method to obtain such an even parity master variable is well known as the Chandrasekhar transformation [26]. Here, we give a short derivation of this transformation, and discuss the metric reconstruction scheme using this new variable ${ }^{(e)} \widetilde{\zeta}$. As mentioned earlier, a part of motivation is the usefulness of master variables which satisfies the same master equation for both the parities. In particular, the master equation is much simpler for the odd parity case. Another point is the appearance of the factor $1 /(\lambda r+3 M)$ in the RW potential in the even parity case, which is absent in the odd parity case. This factor mathematically means the existence of a singularity at $r=-3 M / \lambda$ in the master equation. However, this singularity will not be a physical one because of the symmetry between even and odd parity cases. This factor $1 /(\lambda r+3 M)$ is inherited in many places of the whole reconstruction scheme. Although not a serious obstacle in actual computation, we can expect that the reconstruction scheme might simplify a lot by using the new variable ${ }^{(e)} \widetilde{\zeta}$.

Our quick derivation of ${ }^{(e)} \tilde{\zeta}$ is based on the fact that the Weyl scalar contracted with null tetrad $\psi \equiv-C_{a b c d} l^{a} m^{b} l^{c} m^{d}$ satisfies the same homogeneous equation irrespective of the parity [29]. Here $l^{a}$ and $m^{a}$ are outgoing and angular null tetrad vectors, respectively. For explicit calculations, we use $\left(l^{a}\right)=(r-2 M)^{-1}(r, r$ $-2 M, 0,0)$, and $\left(m^{a}\right)=(\sqrt{2} r \sin \theta)^{-1}(0,0, \sin \theta, i)$. The following formulas are obtained just by plugging in the explicit metric form into the definition of $\psi$ :

$$
\begin{aligned}
{ }^{(o)} \psi= & \frac{i}{2 r^{2}(r-2 M)}\left[(r-2 M) h_{1, r}^{R W}+r h_{0, r}^{R W}-i \omega r h_{1}^{R W}\right. \\
& \left.+\frac{\left(2 M+i \omega r^{2}\right)}{r-2 M} h_{0}^{R W}\right],
\end{aligned}
$$

and

$$
{ }^{(e)} \psi=-\frac{\left(H_{1}^{R W}+H_{2}^{R W}\right)}{r(r-2 M)} .
$$

Here the angular dependence, which is given by the spin weighted spherical harmonics, is suppressed for brevity. We use the same notation ${ }^{(i)} \psi$ to represent the coefficients of Fourier harmonic decomposition, but it will not cause any confusion.

Substituting Eqs. (2.17) and (2.18), we can rewrite Eq. (3.1) in vacuum as

$$
\begin{aligned}
{ }^{(o)} \psi= & \frac{2}{r^{3}(r-2 M)^{2}}\left[\left\{\omega^{2} r^{4}+i \omega r^{2}(r-3 M)\right.\right. \\
& +(3 M-(\lambda+1) r)(r-2 M)\}^{(o)} \zeta(r) \\
& \left.+r\left(i \omega r^{2}+3 M-r\right)(r-2 M)^{(o)} \zeta_{, r}(r)\right] .
\end{aligned}
$$

Here we have used the field equations for simplification. From the equation above and with the aid of Eq. (2.15) in vacuum, we can express the master variable ${ }^{(o)} \zeta$ in terms of ${ }^{(o)} \psi$ and its derivative as

$$
\begin{aligned}
{ }^{(o)} \zeta(r)= & \zeta\left[{ }^{(o)} \psi\right]=\frac{r^{2}}{2(3 i \omega M+\lambda(\lambda+1))}\left[\left\{\omega^{2} r^{3}-5 i \omega M r\right.\right. \\
& -4 M+r+\lambda(2 M-r)\}^{(o)} \psi(r)-(r-2 M)\left(i \omega r^{2}\right. \\
& \left.+3 M-r)^{(o)} \psi_{, r}(r)\right] .
\end{aligned}
$$

Then, with an arbitrary constant $\mathcal{C}$,

$$
\text { (e) } \widetilde{\zeta}=\mathcal{C} \zeta\left[{ }^{(e)} \psi\right]
$$

should satisfy the RW equation, i.e., the same equation that ${ }^{(o)} \zeta$ satisfies except for the source term. After a straightforward calculation, we obtain

$$
\text { (e) } \widetilde{\zeta}(r)=2(r-2 M)\left(H_{2}^{R W}-r K_{, r}^{R W}+\frac{r \lambda}{(r-2 M)} K^{R W}\right),
$$

with

$$
\mathcal{C}=4(3 i \omega M+\lambda(1+\lambda)),
$$


which is known as the Starobinsky constant [27]. It is also easy to check directly that this new master variable satisfies the homogeneous RW equation with the same $V$ as the usual RW potential.

In a general case with the source term, we have

$$
\left[\partial_{r *}^{2}+\omega^{2}-V^{R W}(r)\right]^{(e)} \widetilde{\zeta}=\mathcal{S}^{(e)} \tilde{\zeta}
$$

with

$$
\begin{aligned}
S^{(e) \tilde{\zeta}}= & (r-2 M)\left[-2 r A_{, r}^{(0)}+2 \frac{M-r(1+\lambda)}{r-2 M} A^{(0)}+\sqrt{2} \omega r A^{(1)}+2 \frac{[6 M-r(1+\lambda)](r-2 M)}{r^{2}} A\right. \\
& \left.+2 \frac{[6 M-r(1+\lambda)](r-2 M)}{\sqrt{1+\lambda} r^{2}} B-2 \sqrt{2} \frac{\left[6 M^{2}-\lambda r^{2}(1+\lambda)\right]}{\sqrt{(1+\lambda) \lambda r^{2}}} F-6 \sqrt{2} \frac{r M(r-2 M)}{\sqrt{(1+\lambda) \lambda r}} F_{, r}\right] .
\end{aligned}
$$

The metric reconstruction formulas for the ${ }^{(e)} \widetilde{\zeta}$ are given by

$$
\begin{aligned}
& \hat{K}=\frac{16}{|\mathcal{C}|^{2}}\left[-r(1+\lambda)(r \lambda+3 M) A^{(0)}+\frac{3}{r} \sqrt{1+\lambda} M(r-2 M)^{2}\{B+\sqrt{1+\lambda} A\}-\frac{3}{\sqrt{2}} \omega r M(r-2 M) A^{(1)}\right. \\
& -3 \frac{\sqrt{2(1+\lambda)} M(r-2 M)(r \lambda+3 M)}{r \sqrt{\lambda}} F+\frac{[(1+\lambda)\{3 M(r-2 M)+r \lambda(r \lambda+3 M)\}-r \mathcal{O}]}{2 r^{3}}(e) \widetilde{\zeta} \\
& \left.+\frac{(r \lambda+3 M)(r-2 M)(1+\lambda)}{2 r^{2}}(e) \widetilde{\zeta}_{, r}\right] \text {, } \\
& \hat{H}_{1}=\frac{16}{|\mathcal{C}|^{2}}\left[-i \omega \frac{\mathcal{P} r^{2}(r \lambda+3 M)}{(r-2 M)} A^{(0)}+i \frac{\left[3 M \omega^{2} r+\lambda^{2}(\lambda+1)\right] r^{2}}{\sqrt{2}} A^{(1)}+3 i \omega M \mathcal{P}(r-2 M)\left\{A+\frac{B}{\sqrt{1+\lambda}}\right\}\right. \\
& \left.-3 \sqrt{2} i \omega \frac{M \mathcal{P}(r \lambda+3 M)}{\sqrt{(1+\lambda) \lambda}} F+i \omega \frac{\left[r^{2} \mathcal{O}+3 M \mathcal{P}(r-2 M)\right]}{2 r^{2}(r-2 M)}(e) \tilde{\zeta}+i \omega \frac{(r \lambda+3 M) \mathcal{P}}{2 r}(e) \widetilde{\zeta}_{, r}\right], \\
& \hat{H}_{2}=\frac{16}{|\mathcal{C}|^{2}}\left[\frac{r^{2} \mathcal{O}}{(r-2 M)} A^{(0)}+\omega \frac{\lambda r^{2} \mathcal{P}}{\sqrt{2}} A^{(1)}-\lambda(r-2 M) \mathcal{P}\{(1+\lambda) A+\sqrt{1+\lambda} B\}+\sqrt{2(1+\lambda) \lambda} \mathcal{P}(r \lambda+3 M) F\right. \\
& \left.+\frac{\left[\mathcal{O}-\lambda\left(\omega^{2} r^{3}+M(1+\lambda)\right)\right] \mathcal{P}}{2(r-2 M) r^{2}} \widetilde{\zeta}-\frac{\mathcal{O}}{2 r}(e) \widetilde{\zeta}_{, r}\right], \\
& \hat{H}_{0}=\bar{H}_{2}+\frac{16 \pi}{\sqrt{2 \lambda(1+\lambda)}} r^{2} F \text {. }
\end{aligned}
$$

Here, $\mathcal{P}=3 M-r(1+\lambda)$ and $\mathcal{O}=3 M \omega^{2} r^{2}+\lambda(\lambda+1)(3 M$ $-r$ ). If we are working in frequency domain only, the above choice of master variable is not a bad one because of the common potential in master equation, whereas, in the time domain we will need time integrations for the metric reconstruction due to the factor $|\mathcal{C}|^{-2}$.

We can modify the master variable by adding combinations of metric components which appear on the left-hand side of the Einstein equations. Let us denote these combinations by $G_{(i)}$ so that the Einstein equations are formally written as $G_{(i)}=T_{(i)}(i=1,2, \ldots, 7)$, where $T_{(i)}$ represents each component of the energy momentum tensor, $\left\{A^{(1)}, A, B, A^{(0)}, B^{(0)}, G^{(s)}, F\right\}$. Since $G_{(i)}$ vanishes identically outside the source, the homogeneous equation for the modified master variable should be unaltered by the transformation,

$$
(e) \widetilde{\zeta} \rightarrow{ }^{(e)} \widetilde{\zeta}+\sum_{i=1}^{7} c_{i} G_{(i)}
$$

Now one may think that the factor $|\mathcal{C}|^{-2}$ from the expression for $\hat{h}^{(T)}$ can be eliminated by using this degree of freedom of modifying the master variable. However, we will prove below that it is impossible. 
As a result of the transformation above, $\hat{K}^{(T)}$ is modified as

$$
\hat{K}^{(T)} \rightarrow \hat{K}^{(T)}-\hat{K}^{(M)}\left[\sum_{i=1}^{7} c_{i} T_{(i)}\right]
$$

Since $\hat{K}^{(M)}\left[{ }^{(e)} \widetilde{\zeta}\right]$ contains ${ }^{(e)} \widetilde{\zeta}_{, r}$, we cannot eliminate $T_{(i), r}$ from $\hat{K}^{(M)}\left[\Sigma c_{i} T_{(i)}\right]$ unless $c_{i}=0$ for $i \geqslant 4$. For $\left\{A^{(1)}, A, B\right\}$, one can use the conservation law (2.34) to eliminate $T_{(i), r}$. Thus the condition that $|\mathcal{C}|^{2} \hat{K}^{(M)}\left[\Sigma c_{i} T_{(i)}\right] \approx 0$ requires $c_{i}$ $\approx 0$ for $i \geqslant 4$, where $\approx$ means the equality modulo $|\mathcal{C}|^{2}$. Then, we find that $B^{(0)}$ and $G^{(s)}$ arises in the expression for the modified $\hat{K}^{(T)}$ only from $\Sigma_{i \leqslant 3} c_{i} T_{(i), r}$. Hence, the conditions for the coefficients of $B^{(0)}$ and $G^{(s)}$ to vanish modulo $|\mathcal{C}|^{2}$ become $c_{1} \approx-\left(\omega r^{2} / \sqrt{2(1+\lambda)}(r-2 M)\right) c_{3}$, and $c_{2}$ $\approx \sqrt{(1+\lambda)} c_{3}$, respectively. Thus a possible modification which might eliminate the factor $|\mathcal{C}|^{-2}$ from the expression for $\hat{h}^{(T)}$ is restricted to

$$
\begin{aligned}
\hat{K}^{(T)} \rightarrow & \hat{K}^{(T)}-\hat{K}^{(M)}\left[f ( r ) \left(-\left[\omega r^{2} / \sqrt{2(1+\lambda)}(r-2 M)\right] A^{(1)}\right.\right. \\
& +\sqrt{(1+\lambda)} A+B)]
\end{aligned}
$$

with an arbitrary function $f(r)$. Then, a straightforward calculation shows that the factor $|\mathcal{C}|^{-2}$ cannot be eliminated by this transformation. Thus, the idea of introducing a new master variable for even parity modes satisfying the RW equation does not work well for the purpose of metric reconstruction in the time domain.

\section{DISCUSSION}

In this paper we have introduced new master variables for the odd and the even parity cases. We call them, respectively, the modified Regge-Wheeler and Zerilli variables. These variables satisfy the same Regge-Wheeler or Zerilli equation except for the source terms, which are composed of the matter energy momentum tensor. We have given the explicit expressions for the source term. The metric perturbation in the RW gauge is expressed in terms of the master variables and the matter energy momentum tensor. The explicit formulas for the metric reconstruction were also written down. The important aspect of these modified variables lies in the fact that the frequency $\omega$ does not appear in the denominator in all the formulas to obtain the metric perturbation. Hence, there is no time integration except for the step solving the master equation. The most crucial point will be that $\hat{h}^{(T)}$, the contribution to the reconstructed metric perturbation from the matter energy-momentum tensor, does not have $\omega$ in the denominator. Therefore, the perturbed metric around a field point $(t, r)$ is solely written in terms of the master variables if the energy-momentum tensor vanishes in the vicinity of the spherical shell containing this field point. This fact will be useful in the program to calculate the regularized selfforce acting on a particle orbiting in the Schwarzschild spacetime.

As mentioned earlier, in the Introduction, the full metric perturbation contains a divergent piece near the particle lo- cation. To obtain a sensible expression for the self-force, we need to subtract the so-called "direct" part from the full metric perturbation before evaluating the expression of the force. But here is the "gauge problem." The full metric perturbation is obtained in Regge-Wheeler gauge but the "direct" part is evaluated in the harmonic gauge associated with the particle trajectory.

Here we would like to propose an insight towards an alternative method to handle this gauge issue in the case of the Schwarzschild background. The basic idea is inspired by the notion brought by Barack and Ori [7]. They stressed that the trajectory in the perturbed spacetime is gauge invariant although the expression for the self-force depends on the choice of gauge. On the other hand, the metric perturbations reconstructed from this gauge invariant master variables depend on the choice of gauge, but the concepts of the perturbed geometry and hence of the geodesic on it are gauge invariant. Hence, naturally one may expect that the subtraction at the level of gauge invariant master variables is possible.

A sketch of the new method is the following. The "direct" part of the metric perturbation $h^{(S)}$ can be calculated in the harmonic gauge. We can use the recent observation by Detweiler and Whiting [15] that $h^{(S)}$ can be modified so that it satisfies the Einstein equations. Since the method for the harmonic decomposition of the direct part is established by Mino, Nakano and Sasaki [14], the projection of this direct part to the gauge invariant master variable $\zeta^{(S)}$ is possible by using the formulas (2.14) and (2.26). On the other hand, solving the RW equation, we can directly calculate the master variable corresponding to the full metric perturbation, $\zeta^{(f u l l)}$. Then we subtract the direct part $\zeta^{(S)}$ from $\zeta^{(f u l l)}$ to obtain the master variable that corresponds to the tail part, which we denote by $\zeta^{(t a i l)}$. Since both $\zeta^{(S)}$ and $\zeta^{(f u l l)}$ satisfy the RW equation with the same source, their difference $\zeta^{(t a i l)}$ satisfies the homogeneous RW equation. Hence, we can reconstruct the metric perturbation corresponding to the tail part from this regularized master variable $\zeta^{(\text {tail })}$ by applying the formulas $\hat{h}^{(M)}$. At this step the choice of gauge is unimportant as is explained in the paper by Barack and Ori [7]. Since the subtraction of the divergent part is done at the level of the gauge invariant variables, we would like to call this scheme the gauge invariant regularization.

In the new scheme, using the variables introduced in this paper, the part depending on the master variable in the metric reconstruction formulas, $\hat{h}^{(M)}$, does not have $\omega$ in the denominator as well as $\hat{h}^{(T)}$. Hence, when we know the behavior of the master variable corresponding to a homogeneous solution of metric perturbations in the vicinity of a spherical shell, we can reproduce the metric perturbations from the master variable there. If $\hat{h}^{(M)}$ contained $\omega$ in the denominator, the local information of the master variables near the shell were not sufficient to reproduce the metric perturbations. Therefore, the use of the new variables introduced in this paper is crucial for the gauge invariant regularization.

This scheme still has a subtle point which requires further investigation. The method for the reconstruction of the metric perturbation does apply only for a solution of vacuum 
Einstein equations. However, in the actual computation, the direct part $h^{(S)}$ is calculated in a power series expansion with respect to the separation $\xi$ between the source point and the field point, and this expansion must be truncated at a certain order of $\xi$. Then, the truncated direct part does not satisfy the Einstein equations in general. Hence, we need a new invention to bypass this difficulty in order to realize this attractive idea of the gauge invariant regularization. We would like to return to this challenging issue in a future publication.

In Sec. III we discussed the possibility of using a master variable for even parity modes which has the same potential for the master equation as in the case of odd parity modes. Such a variable is obtained by using the Chandrasekhar transformation. We wrote down the explicit definition of this master variable in terms of the metric components, the master equation with the source terms and the metric reconstruction formulas. We found that the metric reconstruction formulas necessarily contain the Starobinsky constant including $\omega$ in the denominator. Therefore, the use of the even parity master variable that has the same homogeneous master equa- tion as in the odd parity case unfortunately turned out not to be advantageous. However, the master variables considered here are limited to those which are related via Chandrasekhar transformation. We expect an even wider class of transformations in which we might find a more suitable variable for the purpose of metric reconstruction.

\section{ACKNOWLEDGMENTS}

We would like to express special thanks to Y. Mino who gave much insight throughout this work. It is also our pleasure to acknowledge H. Nakano, M. Sasaki, N. Sago and participants of the workshop YITP-W-01-16 held at Yukawa Institute, the Radiation Reaction Focus Session and the 5th Capra Ranch meeting at Penn State, for useful discussions related to this work. S.J. acknowledges support under COE program and the Basque Government. This work is supported in part by Grant in aid for Scientific Research of Japanese Ministry of Education, Culture, Sports, Science and Technology No. 14047212.
[1] M. Sasaki, M. Shibata, H. Tagoshi, and T. Tanaka, Prog. Theor. Phys. Suppl. 128, 1 (1997), and references therein.

[2] See S. Mano and E. Takasugi, Prog. Theor. Phys. 97, 213 (1997), and references therein.

[3] C.O. Lousto and R.H. Price, Phys. Rev. D 55, 2124 (1997).

[4] Y. Mino, M. Sasaki, and T. Tanaka, Phys. Rev. D 55, 3457 (1997); Prog. Theor. Phys. Suppl. 128, 373 (1997).

[5] T.C. Quinn and R.M. Wald, Phys. Rev. D 56, 3381 (1997).

[6] Y. Mino, Prog. Theor. Phys. 99, 79 (1998).

[7] L. Barack and Amos Ori, Phys. Rev. D 61, 061502(R) (2000).

[8] C.O. Lousto, Phys. Rev. Lett. 84, 5251 (2000).

[9] S. Detweiler, Phys. Rev. Lett. 86, 1931 (2001).

[10] L. Barack and A. Ori, Phys. Rev. D 64, 124003 (2001).

[11] H. Nakano, Y. Mino, and M. Sasaki, Prog. Theor. Phys. 106, 339 (2001).

[12] L. Barack, Y. Mino, H. Nakano, A. Ori, and M. Sasaki, Phys. Rev. Lett. 88, 091101 (2002).

[13] M.J. Pfenning and E. Poisson, Phys. Rev. D 65, 084001 (2002).

[14] Y. Mino, H. Nakano, and M. Sasaki, Prog. Theor. Phys. 108, 1034 (2003).

[15] S. Detweiler and B.F. Whiting, Phys. Rev. D 67, 024025
(2003).

[16] N. Sago, H. Nakano, and M. Sasaki, this issue, Phys. Rev. D 67, 104017 (2003).

[17] Y. Mino, Phys. Rev. D 67, 084027 (2003).

[18] B.S. Dewitt and R.W. Brehme, Ann. Phys. (N.Y.) 9, 220 (1960).

[19] T. Regge and J.A. Wheeler, Phys. Rev. 108, 1063 (1957).

[20] F.J. Zerilli, Phys. Rev. D 10, 2141 (1974).

[21] P.L. Chrzanowski, Phys. Rev. D 11, 2042 (1975).

[22] R.M. Wald, Phys. Rev. Lett. 41, 203 (1978).

[23] A. Ori, Phys. Rev. D (to be published), gr-qc/0207045.

[24] V. Moncrief, Ann. Phys. (N.Y.) 88, 323 (1974).

[25] U.H. Gerlach and U.K. Sengupta, Phys. Rev. D 19, 2268 (1979).

[26] S. Chandrasekhar and S. Detweiler, Proc. R. Soc. London A344, 441 (1975).

[27] S. Chandrasekhar, The Mathematical Theory of Black Holes (Oxford University Press, Oxford, England, 1983).

[28] R.J. Gleiser, C.O. Nicasio, R.H. Price, and J. Pullin, Phys. Rep. 325, 41 (2000).

[29] S.A. Teukolsky, Astrophys. J. 185, 635 (1973). 